Supporting Information

\title{
Metabolic engineering of Saccharomyces cerevisiae for rosmarinic acid production
}

Mahsa Babaei ${ }^{1}$, Gheorghe M. Borja Zamfir ${ }^{1}$, Xiao Chen ${ }^{1}$, Hanne Bjerre Christensen ${ }^{1}$, Mette Kristensen ${ }^{1}$, Jens Nielsen ${ }^{1,2,3}$, Irina Borodina*1

${ }^{1}$ The Novo Nordisk Foundation Center for Biosustainability, Technical University of Denmark, Kemitorvet Building 220, DK-2800 Kgs. Lyngby, Denmark

${ }^{2}$ Department of Biology and Biological Engineering, Chalmers University of Technology, 412 96, Gothenburg, Sweden

${ }^{3}$ Biolnnovation Institute, Ole Måløes Vej 3, 2200, Copenhagen N, Denmark

*Corresponding Author 
Supplementary Table S1: Optimization of rosmarinic acid (RA) extraction method. .3

Supplementary Table S2: regression statistics for plant-pathway...........................................................

Supplementary Table S3: regression statistics for chimeric-pathway ......................................................

Supplementary Table S4: List of the strains used in this study...............................................................

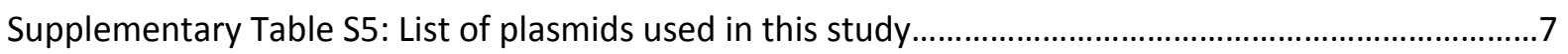

Supplementary Table S6: List of BioBricks used in this study ..............................................................

Supplementary Table S7: List of Primers used in this study................................................................10

Supplementary Table S8: Sequencing Primers used for colony PCR of yeast transformants................11

Supplementary Table S9: Codon-optimized nucleotide sequence of the heterologous genes.............12

Supplementary Figure S1: Stability of rosmarinic acid in mineral media..............................................17

Supplementary Figure S2: Toxicity test of rosmarinic acid on growth of yeast strain...........................18

Supplementary Figure S3: Plasmid maps of integrative vectors............................................................19

Supplementary Figure S4: L-tyrosine degradation to melanin by hydroxylase enzyme EchpaBC.........20

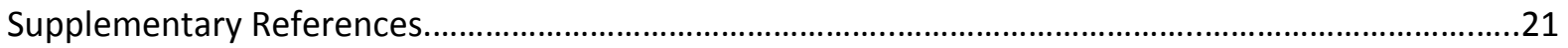


Supplementary Table S1: Optimization of rosmarinic acid (RA) extraction method, with results showing the recovery of analyte. The quantification of RA content in samples was done by LC-MS using freshly-made standards of RA in ethanol. The extraction method 4 was chosen and applied through the current study.

\begin{tabular}{lll}
\hline Extraction Method & Reference & Result \\
\hline $\begin{array}{l}\text { 1) Acidified samples mixed with ethyl acetate for } 5 \\
\text { minutes, the ethyl acetate fraction separated and } \\
\text { evaporated, the pellets re-suspended in methanol. }\end{array}$ & 1,2 & Failed. 0\% recovery of RA \\
$\begin{array}{l}\text { 2) Extraction with acetonitrile, samples light protected. } \\
\begin{array}{l}\text { 3) Mixing the samples with ethanol (volumetric ratio 1:1), } \\
\text { samples light protected. }\end{array}\end{array}$ & This work & Failed. 1\% recovery of RA \\
$\begin{array}{l}\text { 4) Mixing the samples with ethanol containing 0.01\% w/v } \\
\text { butylated hydroxytoluene (BHT), samples light protected. }\end{array}$ & This work & $80-90 \%$ recovery of RA \\
\hline
\end{tabular}

As a major prerequisite to analyze RA, we needed an extraction method with high analytical recovery and precision. To do this, we prepared samples containing low concentration range of RA in mineral medium (10 - $400 \mu \mathrm{g} / \mathrm{L}$ ), followed by extraction of RA and measurements by LC-MS, using calibration curve derived from freshly made standards of RA in ethanol. Based on literature survey, the first method we used, was the previously reported liquid-liquid extraction by ethyl acetate for RA producing E. coli cells ${ }^{1}$. However, by implementing this method with an extraction time of 5 minutes no RA was detected in the extracted samples (Supplementary Table S5). The change in the solvent from ethyl acetate to a more-polar solvent of acetonitrile and protecting the samples from light, resulted in detection of only $4 \mu \mathrm{g} / \mathrm{L}$ rosmarinic acid in the standard sample of $400 \mu \mathrm{g} / \mathrm{L}(1 \%$ recovery). These results ensured us that the efficiency of applied liquid-liquid extraction in aqueous solution is very low, as this method is originally developed for RA extraction from plants ${ }^{1}$. The difference might also be due to different (shorter) extraction time that we applied, as the duration of solvent mixing was not reported in original reference ${ }^{1}$. The solid matrix of the plants is a barrier for mass transfer, which means that the same method might not be applicable to yeast cultures. Therefore, we simplified the sample preparation procedure, and proceeded with RA quantification in aqueous phase. As RA solubility is reported to increase in binary solvent mixture of ethanol and water, so the aqueous samples were mixed with ethanol in a volumetric ratio of 1:1. By using this method for sample preparation, the recovery was significantly increased to $80-90 \%$. Furthermore, to inhibit spontaneous oxidation of rosmarinic acid, $0.01 \% \mathrm{w} / \mathrm{v}$ of butylated hydroxytoluene (BHT) was supplemented to ethanol, as this modification is shown to prevent spontaneous oxidation of resveratrol; a similar-structured aromatic compound ${ }^{3}$. This method led to over $99 \%$ recovery of rosmarinic acid in the samples (Supplementary Table S5), which was used as the extraction method in all of the samples throughout the rest of the study. 
Supplementary Table S2: regression statistics for plant-pathway $2^{3} \mathrm{DOE}$

\begin{tabular}{lcccc} 
Source & SS $_{\mathrm{B}}{ }^{*}$ & F-value & p-value & Remarks \\
\hline Model & 5.27614 & 77.01 & 0.087 & Not significant \\
Linear & 2.50335 & 73.07 & 0.086 & Not significant \\
RAS & 1.98999 & 174.26 & 0.048 & Significant \\
TAT & 0.41367 & 36.23 & 0.105 & Not significant \\
CYP-CPR & 0.09969 & 8.73 & 0.208 & Not significant \\
2-Way Interactions & 2.77279 & 80.94 & 0.081 & Not significant \\
RAS*TAT & 1.43946 & 126.05 & 0.057 & Not significant \\
RAS $^{*}$ CYP-CPR & 1.10918 & 97.13 & 0.064 & Not significant \\
TAT ${ }^{*}$ CYP-CPR & 0.22415 & 19.63 & 0.141 & Not significant
\end{tabular}

${ }^{*} \mathrm{SS}_{\mathrm{B}}$ : sum of squares between groups, indicating the interaction between the factors.

Supplementary Table S3: regression statistics for chimeric-pathway $2^{2} \mathrm{DOE}$

\begin{tabular}{lcccc} 
Source & SS $_{\mathbf{B}}$ & F-value & p-value & Remarks \\
\hline Model & 0.04081 & 1.88 & 0.458 & Not significant \\
Linear & 0.04081 & 1.88 & 0.458 & Not significant \\
RAS & 0.01396 & 1.29 & 0.460 & Not significant \\
TAT & 0.02685 & 2.48 & 0.360 & Not significant
\end{tabular}


Supplementary Table S4: List of the strains used in this study

\begin{tabular}{|c|c|c|c|c|}
\hline Name & $\begin{array}{l}\text { Parental } \\
\text { Strain }\end{array}$ & $\begin{array}{l}\text { Added DNA } \\
\text { Element(s) }\end{array}$ & Relevant Genotype & Source \\
\hline $\begin{array}{l}\text { CEN.PK } \\
113-7 D\end{array}$ & & & MATa MAL2-8c SUC2 URA3 HIS3 LEU2 TRP1 & $\begin{array}{l}\text { Peter } \\
\text { Kötter }\end{array}$ \\
\hline ST7574 & $\begin{array}{l}\text { CEN.PK } \\
\text { 113-7D }\end{array}$ & pCfB2312 & CEN.PK113-7D 个Cas9_KanMX & 4 \\
\hline ST9155 & ST7574 & pCfB1071, pCfB0975 & $\begin{array}{l}\text { CEN.PK113-7D } \uparrow C a s 9 \_K a n M X ~ \uparrow C b R A S ~ \uparrow C b H P P R \\
\uparrow C b C Y P \uparrow C b C P R\end{array}$ & This study \\
\hline ST9156 & ST7574 & pCfB1071, pCfB0976 & $\begin{array}{l}\text { CEN.PK113-7D } \text { CCas9_KanMX } \uparrow C b R A S \uparrow C b H P P R \\
\text { 个SmCYP } \text { 个SmCPR }\end{array}$ & This study \\
\hline ST9157 & ST7574 & pCfB1072, pCfB0975 & $\begin{array}{l}\text { CEN.PK113-7D 个Cas9_KanMX 个MoRAS 个CbHPPR } \\
\text { 个CbCYP 个CbCPR }\end{array}$ & This study \\
\hline ST9158 & ST7574 & pCfB1072, pCfB0976 & 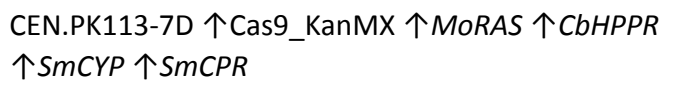 & This study \\
\hline ST9398 & ST9155 & PR-17720, PR-16208 & $\begin{array}{l}\text { CEN.PK113-7D 个Cas9_KanMX 个CbRAS 个CbHPPR } \\
\text { 个CbCYP } \uparrow C b C P R \triangle p d c 5 \Delta a r o 10\end{array}$ & This study \\
\hline ST9399 & ST9156 & PR-17720, PR-16208 & $\begin{array}{l}\text { CEN.PK113-7D 个Cas9_KanMX 个CbRAS 个CbHPPR } \\
\text { 个SmCYP 个SmCPR } \triangle p d c 5 \Delta a r o 10\end{array}$ & This study \\
\hline ST9400 & ST9157 & PR-17720, PR-16208 & $\begin{array}{l}\text { CEN.PK113-7D 个Cas9_KanMX 个MoRAS 个CbHPPR } \\
\text { 个CbCYP 个CbCPR } \triangle p d c 5 \Delta \text { aro10 }\end{array}$ & This study \\
\hline ST9401 & ST9158 & PR-17720, PR-16208 & 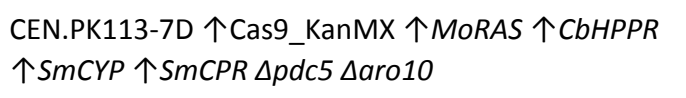 & This study \\
\hline ST9402 & ST7574 & PR-17720, PR-16208 & CEN.PK113-7D 个Cas9_KanMX $\Delta p d c 5 \Delta a r o 10$ & This study \\
\hline ST9403 & ST9398 & $\begin{array}{l}\text { pCfB9114, pCfB9145, } \\
\text { pCfB9146 }\end{array}$ & 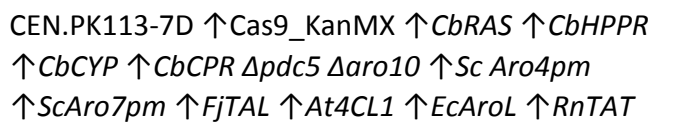 & This study \\
\hline ST9404 & ST9399 & $\begin{array}{l}\text { pCfB9114, pCfB9145, } \\
\text { pCfB9146 }\end{array}$ & 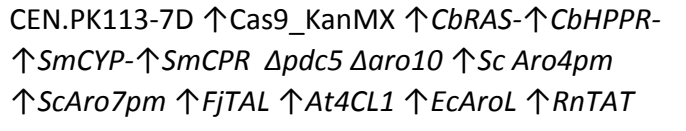 & This study \\
\hline ST9405 & ST9400 & $\begin{array}{l}\text { pCfB9114, pCfB9145, } \\
\text { pCfB9146 }\end{array}$ & 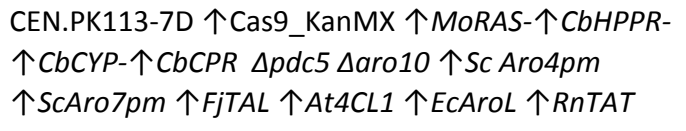 & This study \\
\hline ST9406 & ST9401 & $\begin{array}{l}\text { pCfB9114, pCfB9145, } \\
\text { pCfB9146 }\end{array}$ & 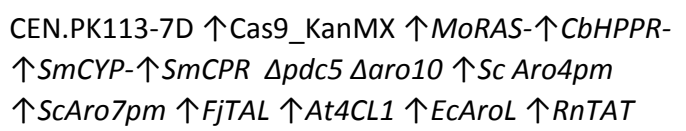 & This study \\
\hline
\end{tabular}




\begin{tabular}{|c|c|c|c|c|}
\hline ST9407 & ST9402 & $\begin{array}{l}\text { pCfB9114, pCfB9145, } \\
\text { pCfB9146 }\end{array}$ & $\begin{array}{l}\text { CEN.PK113-7D 个Cas9_KanMX } \triangle p d c 5 \Delta \text { aro } 10 \uparrow S c \\
\text { Aro4pm 个ScAro7pm 个FjTAL 个At4CL1 个EcAroL } \\
\text { 个RnTAT }\end{array}$ & This study \\
\hline ST9408 & ST9402 & $\begin{array}{l}\text { pCfB9114, pCfB9145, } \\
\text { pCfB9147 }\end{array}$ & 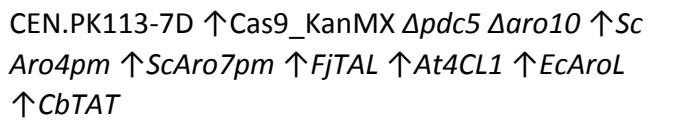 & This study \\
\hline ST9409 & ST9398 & $\begin{array}{l}\text { pCfB9114, pCfB9145, } \\
\text { pCfB9147 }\end{array}$ & 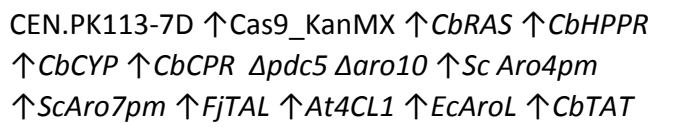 & This study \\
\hline ST9410 & ST9399 & $\begin{array}{l}\text { pCfB9114, pCfB9145, } \\
\text { pCfB9147 }\end{array}$ & 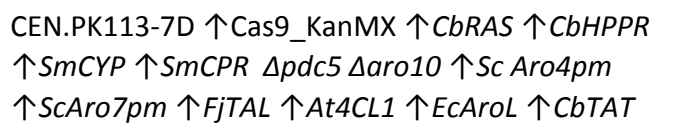 & This study \\
\hline ST9411 & ST9400 & $\begin{array}{l}\text { pCfB9114, pCfB9145, } \\
\text { pCfB9147 }\end{array}$ & 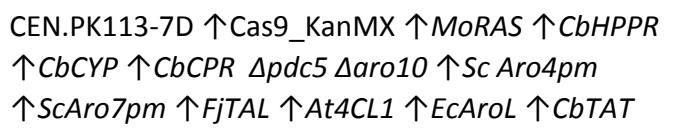 & This study \\
\hline ST9412 & ST9401 & $\begin{array}{l}\text { pCfB9114, pCfB9145, } \\
\text { pCfB9147 }\end{array}$ & 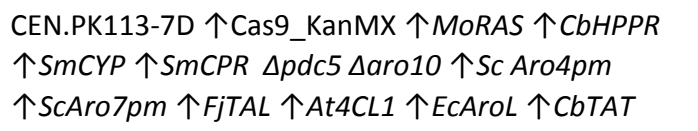 & This study \\
\hline ST9413 & ST9407 & pCfB1071, pCfB2771 & 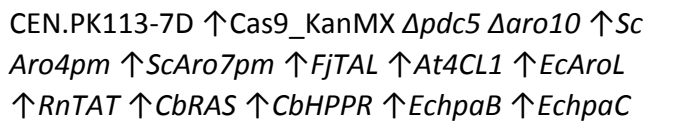 & This study \\
\hline ST9414 & ST9407 & pCfB1072, pCfB2771 & 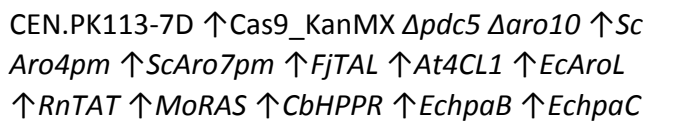 & This study \\
\hline ST9415 & ST9408 & pCfB1071, pCfB2771 & 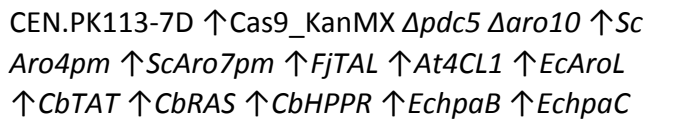 & This study \\
\hline ST9416 & ST9408 & pCfB1072, pCfB2771 & 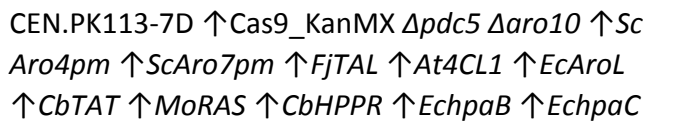 & This study \\
\hline ST9522 & ST9405 & pCfB9217, pCfB9220 & 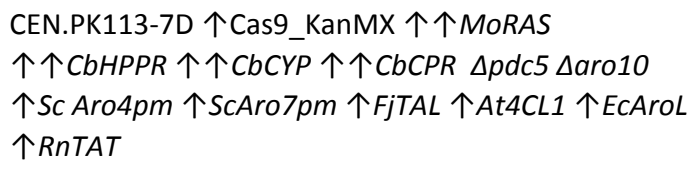 & This study \\
\hline ST9526 & ST9411 & pCfB9217, pCfB9220 & 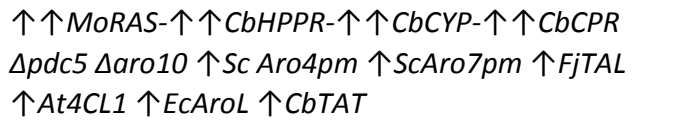 & This study \\
\hline ST9527 & ST9412 & pCfB9218, pCfB9220 & 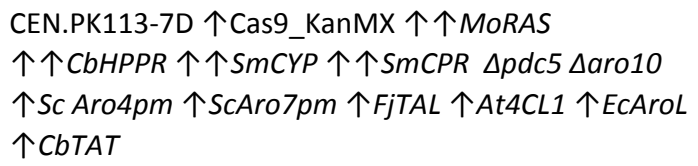 & This study \\
\hline
\end{tabular}


Supplementary Table S5: List of plasmids used in this study

\begin{tabular}{|c|c|c|c|}
\hline Plasmid & $\begin{array}{l}\text { Parent } \\
\text { Vector }\end{array}$ & BioBricks & Source \\
\hline \multicolumn{4}{|l|}{ Episomal Plasmids } \\
\hline pCfB2312(CEN/ARS_Cas9_kanMX) & & & 5 \\
\hline \multicolumn{4}{|l|}{ gRNA Plasmids } \\
\hline pTAJAK-71 (pESC-NatMXsyn-USER) & & & 6 \\
\hline pCfB3052 (p-gRNA X-4 XI-3 XII-5) & & & 6 \\
\hline pCfB4156 (p-gRNA_PDC5_ARO10) & & & 7 \\
\hline pCfB9014(pgRNA_XI-2_XII-4_Nat) & pTAJAK-71 & $\begin{array}{l}\text { BB3799(XI-2_USER gRNA2 P1R), BB4015(XII- } \\
\text { 4_USER gRNA1) }\end{array}$ & This study \\
\hline pCfB8906(NatMX_gRNA_X-3 XII-2) & pTAJAK-71 & $\begin{array}{l}\text { BB3960(XII-2_USER gRNA2), BB3961(X-3_USER } \\
\text { gRNA1) }\end{array}$ & This study \\
\hline \multicolumn{4}{|l|}{ Integration Plasmids } \\
\hline pCfB2903(XI-2-MarkerFree) & & & 6 \\
\hline pCfB2904(XI-3-MarkerFree) & & & 6 \\
\hline pCfB2909(XII-5-MarkerFree) & & & 6 \\
\hline pCfB3034 (X-3-MarkerFree) & & & 6 \\
\hline pCfB3035 (X-4-MarkerFree) & & & 6 \\
\hline pCfB3039 (XII-2-MarkerFree) & & & 6 \\
\hline pCfB3040 (XII-4-MarkerFree) & & & 6 \\
\hline pCfB975(pXII-4<-CbCYP98A14-CbCPR->) & pCfB3040 & $\begin{array}{l}\text { BB0010 (<-TEF1:PGK1->), BB0386 } \\
\text { (CbCYP98A14<-), BB0385 (CbCPR->) }\end{array}$ & This study \\
\hline pCfB976(pXII-4<-SmCYP98A14-SmCPR->) & pCfB3040 & $\begin{array}{l}\text { BB0010 (<-TEF1:PGK1->), BB0392 } \\
(\text { SmCYP98A14<-), BB0391 (SmCPR->) }\end{array}$ & This study \\
\hline pCfB1071(pXI-2<-CbRAS-CbHPPR->) & pCfB2903 & $\begin{array}{l}\text { BB0010 (<-TEF1:PGK1->), BB0387 (CbRAS<-), } \\
\text { BB0384 (CbHPPR->) }\end{array}$ & This study \\
\hline pCfB1072(pXI-2<-MoRAS-CbHPPR->) & pCfB2903 & $\begin{array}{l}\text { BB0010 (<-TEF1:PGK1->), BB0389 (MoRAS<-), } \\
\text { BB0384 (CbHPPR->) }\end{array}$ & This study \\
\hline pCfB2771(XII-4_<-EchpaB-EchpaC->) & pCfB3040 & $\begin{array}{l}\text { BB0010 (<-TEF1:PGK1->), BB0585 (EchpaB<-), } \\
\text { BB0586 (EchpaC->) }\end{array}$ & This study \\
\hline
\end{tabular}




\begin{tabular}{|l|l|l|l|}
\hline pCfB9114(X-4<-SCAro4pm-ScAro7pm->) & pCfB3035 & $\begin{array}{l}\text { BB0010 (<-TEF1:PGK1->), } \\
\text { BB4021(ARO4K229L_U1), } \\
\text { BB4083(ARO7G141S_U2) }\end{array}$ & This study \\
\hline pCfB9145 (XI-3<-FjTAL-At4CL1->) & pCfB2904 & $\begin{array}{l}\text { BB0010 (<-TEF1:PGK1->), BB0380 (FjTAL<-), } \\
\text { BB4110 (At4CL1_U2) }\end{array}$ & This study \\
\hline pCfB9146 (XII-5 <-ECAroL-RnTAT->) & pCfB2909 & $\begin{array}{l}\text { BB0010 (<-TEF1:PGK1->), BB4111 (aroL_U1), } \\
\text { BB0390 (RnTAT->) }\end{array}$ & This study \\
\hline pCfB9147 (XII-5 <-ECAroL-CbTAT->) & pCfB2909 & $\begin{array}{l}\text { BB0010 (<-TEF1:PGK1->), BB4111 (aroL_U1), } \\
\text { BB0388 (CbTAT->) }\end{array}$ & This study \\
\hline pCfB9217 (pX-3<-CbCYP98A14-CbCPR->) & pCfB3034 & $\begin{array}{l}\text { BB0010 (<-TEF1:PGK1->), BB0386 } \\
\text { (CbCYP98A14<-), BB0385 (CbCPR->) }\end{array}$ & This study \\
\hline pCfB9218(pX-3<-SmCYP98A14-SmCPR->) & pCfB3034 & $\begin{array}{l}\text { BB0010 (<-TEF1:PGK1->), BB0392 } \\
\text { (SmCYP98A14<-), BB0391 (SmCPR->) }\end{array}$ & This study \\
\hline pCfB9219 (pXII-2<-CbRAS-CbHPPR->) & pCfB3039 & $\begin{array}{l}\text { BB0010 (<-TEF1:PGK1->), BB0387 (CbRAS<-), } \\
\text { BB0384 (CbHPPR->) }\end{array}$ & This study \\
\hline
\end{tabular}

Supplementary Table S6: List of BioBricks used in this study

\begin{tabular}{|c|c|c|c|c|}
\hline BioBrick & PCR template & Forward primer & Reverse primer & Source \\
\hline BB0010 (<-TEF1:PGK1->) & pCfB0029 & PR-1750 & PR-8 & 7 \\
\hline BB0380 (FjTAL<-) & & PR-1691 & PR-1692 & 7 \\
\hline BB0384 (CbHPPR->) & p0793 & PR-1721 & PR-1722 & This study \\
\hline BB0385 (CbCPR->) & p0805 & PR-1723 & PR-1724 & This study \\
\hline BB0386 (CbCYP98A14<-) & p0806 & PR-1725 & PR-1726 & This study \\
\hline BB0387 (CbRAS<-) & p0807 & PR-1727 & PR-1728 & This study \\
\hline BB0388 (CbTAT->) & p0808 & PR-1729 & PR-1730 & This study \\
\hline BB0389 (MoRAS<-) & p0809 & PR-1731 & PR-1732 & This study \\
\hline BB0390 (RnTAT->) & p0810 & PR-1733 & PR-1734 & This study \\
\hline BB0391 (SmCPR->) & p0811 & PR-1735 & PR-1736 & This study \\
\hline BB0392 (SmCYP98A14<-) & p0812 & PR-1737 & PR-1738 & This study \\
\hline BB0585 (EchpaB<-) & p2120 & PR-7334 & PR-7335 & This study \\
\hline BB0586 (EchpaC->) & p2121 & PR-7336 & PR-7337 & This study \\
\hline $\begin{array}{l}\text { BB3799(XI-2_USER gRNA2 } \\
\text { P1R) }\end{array}$ & pCfB3044 (p-gRNA XI-2) & PR-10526 & PR-10529 & This study \\
\hline BB3960(XII-2_USER gRNA2) & pCfB3048 (p-gRNA XII-2) & PR-10526 & PR-10529 & This study \\
\hline BB3961(X-3_USER gRNA1) & pCfB3041 (p-gRNA X-3) & PR-10525 & PR-10530 & This study \\
\hline
\end{tabular}




\begin{tabular}{|l|l|l|l|l|}
\hline BB4015(XII-4_USER gRNA1) & pCfB3049 (p-gRNA XII-4) & PR-10525 & PR-10530 & This study \\
\hline BB4021(ARO4K229L_U1) & p2817 & PR-1388 & PR-1389 & This study \\
\hline BB4083(ARO7G141S_U2) & p2818 & PR-1390 & PR-1391 & This study \\
\hline BB4110 (At4CL1_U2) & p0757 & PR-24928 & PR-24929 & This study \\
\hline BB4111 (aroL_U1) & pCfB4289 & PR-24930 & PR-24931 & This study \\
\hline
\end{tabular}


Supplementary Table S7: List of Primers used in this study. The USER overhang sequence is underlined

\begin{tabular}{|c|c|}
\hline Primer & Sequence $\left(5^{\prime}->3^{\prime}\right)$ \\
\hline PR-8 (PPGK1_rv) & ATGACAGAUTTGTTTTATATTTGTTTG \\
\hline PR-16208 (PDC5_KO_OLIGO) & $\begin{array}{l}\text { ATGTCTGAAATAACCTTAGGTAAATATTTATTTGAAAGATTGAGCGTTAAACAA } \\
\text { GCTCAATTGACTGCCGCTACTAACGCTAAACAATAA }\end{array}$ \\
\hline PR-17720 (ARO10_KO_OLIGO) & $\begin{array}{l}\text { ATGGCACCTGTTACAATTGAAAAGTTCGTAAATCAAGAAGAACGAAAGTGCAT } \\
\text { GGTTGAAGCAGCGGCACTTAAAAGAAATAAAAAATAG }\end{array}$ \\
\hline PR-1691 (FjTAL_U1_fw) & AGTGCAGGUAAAACAATGAACACCATCAACGAATATCTGAGC \\
\hline PR-1692 (FjTAL_U1_rv) & CGTGCGAUTTAATTGTTAATCAGGTG \\
\hline PR-1721 (CbHPPR_U2_fw) & ATCTGTCAUAAAACAATGGAAGCCATCG \\
\hline PR-1722 (CbHPPR_U2_rv) & CACGCGAU TCAGACAACTGGTGTCAACAAAG \\
\hline PR-1723 (CbCPR_U2_fw) & ATCTGTCAUAAAACAATGGAATCCACCATC \\
\hline PR-1750 (PTEF1_fw) & ACCTGCACUTTGTAATTAAAACTTAGATTAGATTG \\
\hline PR-1724 (CbCPR_U2_rv) & CACGCGAU TCACCAAACATCTCTTAAGACTG \\
\hline PR-1725 (CbCYP98A14_U1_fw) & AGTGCAGGUAAAACAATGGCTTCCCCTTTG \\
\hline PR-1726 (CbCYP98A14_U1_rv) & CGTGCGAUTCAAATATCAACAACGGCAACTC \\
\hline PR-1727 (CbRAS_U1_fw) & AGTGCAGGUAAAACAATGAAGATCGAAGTTAAGG \\
\hline PR-1728 (CbRAS_U1_rv) & CGTGCGAUTCAAATTTCGTAGAACAACTTTTCG \\
\hline PR-1729 (CbTAT_U2_fw) & ATCTGTCAUAAAACAATGGAATTGCAAAACTC \\
\hline PR-1730 (CbTAT_U2_rv) & CACGCGAUTCAGTAAGAGTGTCTGATGCAGAAG \\
\hline PR-1731 (MoRAS_U1_fw) & AGTGCAGGUAAAACAATGAGAATCGACATCAAG \\
\hline PR-1732 (MoRAS_U1_rv) & CGTGCGAUTCAAATTTCGTAGAACAACTTTTCG \\
\hline PR-1733 (RnTAT_U2_fw) & ATCTGTCAUAAAACAATGGTTTCCTACGTTATC \\
\hline PR-1734 (RnTAT_U2_rv) & CACGCGAUTCACTTATCACATTCTTCTTGAGAAC \\
\hline PR-1735 (SmCPR_U2_fw) & ATCTGTCAUAAAACAATGGAACCATCCTC \\
\hline PR-1736 (SmCPR_U2_rv) & CACGCGAUTCACCAGACATCTCTCAAGTATC \\
\hline PR-1737 (SmCYP98A14_U1_fw) & AGTGCAGGUAAAACAATGGCTGCTTTGTTG \\
\hline PR-1738 (SmCYP98A14_U1_rv) & CGTGCGAUTCAGATGGTGTCAACAGCAAC \\
\hline PR-7334(EcHPAB_U1_fw) & AGTGCAGGUAAAACAATGAAGCCAGAAG \\
\hline PR-7335(EcHPAB_U1_rv) & CGTGCGAUTTACTTCAACAACTTGTCCAAC \\
\hline PR-7336 (EcHPAC_U2_fw) & ATCTGTCAUAAAACAATGCAATTGGAC \\
\hline PR-7337 (EcHPAC_U2_rv) & CACGCGAUTCAAATAGCAGCTTCCAT \\
\hline PR-24928 (At4CL1_U2_fw) & ATCTGTCAU AAAACAATGGCTCCACAAGAACAAGCTGTTTCC \\
\hline PR-24929 (At4CL1_U2_rv) & CACGCGAU TCACAAACCGTTAGCCAA \\
\hline PR-24930 (aroL_U1_fw) & AGTGCAGGU AAAACA ATGACACAACCTCTTTTTTCTGA \\
\hline PR-24931 (aroL_U1_rv) & CGTGCGAU TCAACAATTGATCGTCTGTGC \\
\hline PR-1388 (aro4_U1_fw) & AGTGCAGGU AAAACA ATGAGTGAATCTCCAATGTTCG \\
\hline PR-1389 (aro4_U1_rv) & CGTGCGAU TCA TTTCTTGTTAACTTCTCTTCTTTG \\
\hline PR-1390 (aro7_U2_fw) & ATCTGTCAU AAAACA ATGGATTTCACAAAACCAGAAAC \\
\hline PR-1391 (aro7_U2_rv) & CACGCGAU TCA CTCTTCCAACCTTCTTAGCAAG \\
\hline
\end{tabular}




\begin{tabular}{|c|c|}
\hline PR-10525 (TJOS-62 (P1F)) & CGTGCGAUAGGGAACAAAAGCTGGAGCT \\
\hline PR-10526 (TJOS-63 (P2F)) & AGTGCAGGUAGGGAACAAAAGCTGGAGCT \\
\hline PR-10529 (TJOS-65 (P1R)) & CACGCGAUTAACTAATTACATGACTCGA \\
\hline PR-10530 (TJOS-66 (P2R)) & ACCTGCACUTAACTAATTACATGACTCGA \\
\hline PR-24928 (At4CL1_U2_fw) & ATCTGTCAUAAAACAATGGCTCCACAAGAACAAGCTGTTTCC \\
\hline PR-24929 (At4CL1_U2_rv) & CACGCGAUTCACAAACCGTTAGCCAA \\
\hline PR-24930 (aroL_U1_fw) & AGTGCAGGUAAAACA ATGACACAACCTCTTTTTTCTGA \\
\hline PR-24931 (aroL_U1_rv) & CGTGCGAUTCAACAATTGATCGTCTGTGC \\
\hline PR-903 & TGACGAATCGTTAGGCACAG \\
\hline PR-904 & CCGTGCAATACCAAAATCG \\
\hline PR-905 & CTCACAAAGGGACGAATCCT \\
\hline PR-906 & GACGGTACGTTGACCAGAG \\
\hline PR-909 & GTTTGTAGTTGGCGGTGGAG \\
\hline PR-910 & GAGACAAGATGGGGCAAGAC \\
\hline PR-911 & GTGCTTGATTTGCGTCATTC \\
\hline PR-912 & CACATTGAGCGAATGAAACG \\
\hline PR-893 & CGAAGAAGGCCTGCAATTC \\
\hline PR-894 & GGCCCTGATAAGGTTGTTG \\
\hline PR-897 & GAACTGACGTCGAAGGCTCT \\
\hline PR-898 & CGTGAAATCTCTTTGGGGTAG \\
\hline PR-899 & CCACCGAAGTTGATTTGCTT \\
\hline PR-900 & GTGGGAGTAAGGGATCCTGT \\
\hline PR-2221 & GTTGACACTTCTAAATAAGCGAATTTTC \\
\hline
\end{tabular}

Supplementary Table S8: Sequencing Primers used for colony PCR of yeast transformants. The primer 1 and 2 bind to regions to homologous arms of UP and DW of integration site, while PR-2221 (primer 3) binds to Tadh1. Depending on the primers bound to genomic DNA of yeast transformant used as template, the correct or wild-type size of fragment is obtained.

\begin{tabular}{|l|c|c|c|c|c|}
\hline \multirow{2}{*}{ EasyClone Site } & \multicolumn{3}{|c|}{ Primers } & \multicolumn{2}{c|}{ Expected fragment Size } \\
\cline { 5 - 6 } & Primer 1 & Primer 2 & Primer 3 & Correct (bp) & Wild type (bp) \\
\hline chX-3 & PR-903 & PR-904 & PR-2221 & 1059 & 1482 \\
\hline $\operatorname{chX}-4$ & PR-905 & PR-906 & PR-2221 & 983 & 1394 \\
\hline chXI-2 & PR-909 & PR-910 & PR-2221 & 963 & 1573 \\
\hline $\operatorname{chXI-3~}$ & PR-911 & PR-912 & PR-2221 & 927 & 1450 \\
\hline $\operatorname{chXII-2~}$ & PR-893 & PR-894 & PR-2221 & 795 & 1300 \\
\hline $\operatorname{chXII-4~}$ & PR-897 & PR-898 & PR-2221 & 828 & 1248 \\
\hline $\operatorname{chXII-5~}$ & PR-899 & PR-900 & PR-2221 & 811 & 1365 \\
\hline
\end{tabular}


Supplementary Table S9: Codon-optimized nucleotide sequence of the heterologous genes

\begin{tabular}{|c|c|}
\hline $\begin{array}{l}\text { Gene } \\
\text { name }\end{array}$ & Sequence \\
\hline FjTAL & $\begin{array}{l}\text { atgaacaccatcaacgaatatctgagcctggaagaattgaagccattatcttggcaatcagaaag } \\
\text { tgaccattagtgatgttgttgtgaatcgcgttaacgagagctttaactttctgaaagaatttagcggcaa } \\
\text { caaagtgatctatggtgtgaataccggttttggtccgatggcacagtatcgtattaaagaaagcgatc } \\
\text { agattcagctgcagtataatctgattcgtagccatagcagcggcaccggtaaaccgctgagtccggt } \\
\text { ttgtgcaaaagcagcaattctggcacgtctgaataccctgagtctgggtaatagcggtgttcatccga } \\
\text { gcgttattaatctgatgagcgaactgatcaacaaagatatcacaccgctgattttgaacatggtggtg } \\
\text { ttggtgcaaagcggtgatctggttcagctgagccatctggcactggttctgattggtgaaggtgaagtttt } \\
\text { ctataaaggtgaacgtcgtccgacaccggaagttttgaaattgaaggtctgaaaccgatccaggtg } \\
\text { gaaattcgcgaaggtctggccctgattaatggcaccagcgttatgaccggtattggtgttgttaatgtgt } \\
\text { accatgcaaaaaaactgctggattggagcctgaaaagcagctgtgcaattaatgaactggttcagg } \\
\text { catatgatgatcactttagcgcagaactgaatcagaccaaacgtcataaaggtcagcaagaaattg } \\
\text { cactgaaaatgcgtcagaatctgagcgatagcaccctgattcgcaaacgtgaagatcatctgtata } \\
\text { gcggtgaaaacaccgaagaaatcttcaaaagaaaaagtgcaagagtattatagcctgcgttgtgttc } \\
\text { cgcagattctgggtccggttctggaaaccattaacaatgttgcaagcattctggaagatgaatttaac } \\
\text { agcgcaaacgataacccgatcatcgatgttaaaaaccagcatgtttatcacggtggcaattttcatg } \\
\text { gtgattatatcagcctggaaatggataaactgaaaatcgtgattaccaaactgaccatgctggcaga } \\
\text { acgtcagctgaattatctgctgaatagcaaaattaacgaactgctgcctccgtttgttaatctgggcac } \\
\text { cctgggttttaactttggtatgcagggtgttcagtttaccgcaaccagcaccaccgcagaaagccag } \\
\text { atgctgagcaatccgatgtatgttcatagcattccgaacaataatgataaccaggatattgttagcatg } \\
\text { ggcaccaatagcgcagttattaccagcaaagttatcgaaaatgcctttgaagttctggccattgaaat } \\
\text { gattaccattgttcaggcgattgattatctgggccagaaagataaaatcagcagcgttagcaaaaaa } \\
\text { tggtatgatgaaatccgcaacatcatcccgacctttaaagaagatcaggtgatgtatccgttcgtgca } \\
\text { gaaagtaaaagaccacctgattaacaattaa }\end{array}$ \\
\hline CDTAT & $\begin{array}{l}\text { atggaattgcaaaactccgctcaagaaatggaagctccaactactattaccatcaagggtatttgg } \\
\text { gtttgttgatggctaacaccgatgctaaagaaaacggtaagagagttatctccttgggtattggtgatc } \\
\text { caactgcttactcttgtttcatgcttctaatgctgctcaagaaggtgttgttgaatgtttgagatctgctaa } \\
\text { gtttaacggttatgctccaacagctggtttgccacaaactagacaagctattgctgaatacttgtccag } \\
\text { agatttgccatacaaattgccagctgaatctgtttatgttactgctggttgtactcaagccattgaaattg } \\
\text { ctttgtctgttttggctagaccaggtgctaacattttgttgccaagaccatgttttccaatctacggtttgtgt } \\
\text { gcttccttcagaaacatcgaagttagatactttgacttgcacccagaaaaaggttgggaagttgactt } \\
\text { acaagctgttgaagatttggctgatcataacactgttgccatggttattatcaatccaggtaatccatgt } \\
\text { ggtaacgtctactcttaccaacacttgaagaaagttgctgaaaccgctaaaagattgggtatcgttgtt } \\
\text { attgccgatgaagtttatggtcatttggcttttggtgctaatccatttgttccaatgggtaactttggttccatt } \\
\text { gctccagttgttactttgggttctttgtctaaaagatggttggttccaggttggagattgggttggttggttat } \\
\text { caatgatccagatggtacttgatgtccccaaagttgttgaaagaatcaaaaagtactgcgacatttg } \\
\text { tggtggtccagctacttttattcaagctgctgttccagaaatcgttgaacaaactcaagaagttttcttca }\end{array}$ \\
\hline
\end{tabular}


gaaagaccatcaatatcttgagacaaacctccgatatttgctgccaaaagatcgaagaaatcgatg gtattacctgtccaactaagccaaaaggtgctatggcttttatggttaagttgaacatctccagaatga aggatatctccgatgatattgacttctgcttcaagttggccaaagaagaatccgttattatcttgccagg tattgctgttggtttgaacaactggttgagaattactttcgctgttgaaccatctgctttggaagaagctat ggaaagattgaagtccttctgcatcagacactcttactga

RnTAT atggtttcctacgttatccaaaccgatgttgatgactcttgtcctctgtttggatgttcacgttaacattgg tggtagaaactctgttcaaggtagaaagaagggtagaaaagctagatgggatgttagaccatctga catgtctaacaagaccttcaatccaattagagccatcgttgataacatgaaggttcaacctaatccaa acaagaccgtcatctcttgtctattggtgatccaactgtttcggtaacttgccaactgatcctgaagtta ctcaagctatgaaggatgctttggattctggtaagtataatggttacgctccatccattggttacttgtcat ctagagaagaagttgcctcttactaccattgtcatgaagctccattggaagccaaggatgttattttga cttctggttgttcccaagccattgaattgtgtttggctgtttggctaatccaggtcaaaacattttgattcc aagaccaggtttctcattatacagaaccttggctgaatccatgggtatcgaagttaagttgtacaatttg ttgccagaaaagtcctgggaaatcgacttgaaacaattggaatccttgatcgacgaaaagaccgct tgtttggttgtaaacaatccatctaatccatgcggttccgtttttctaaaagacacttgcaaaagattttg gccgttgctgaaagacaatgcgttccaattttggctgacgaaatctatggtgacatggtttttctgactg caagtatgaaccattggctaacttgtctaccaacgttcctatttgtcttgtggtggtttggctaaaagatg gttggttccaggttggagattgggttggatattgattcatgatagaagagacatcttcggtaacgaaat cagagatggtttggtcaagttgtcccaaagaattttgggtccatgtactatagttcaaggtgccttgaaa tccatcttgcaaagaactccacaagaattctaccacgataccttgtctttcttgaagtctaacgctgattt gtgttacggtgcttggctgctattccaggtttacaacctgttagaccttcaggtgctatgtacttaatggtt ggtatcgaaatggaacacttcccagaattcgaaaacgatgttgaattcaccgaaagattgattgctg aacaagctgttcattgcttgccagctacttgtttgaatacccaaacttcttcagagttgttatcactgtac ctgaagtcatgatgttggaagcttgctctagaattcaagaattctgcgaacaacattaccattgcgctg aaggttctcaagaagaatgtgataagtga

At4CL 1 atggctccacaagaacaagctgtttcccaagttatggaaaagcaatctaacaacaacaactccga cgtcatcttcagatctaaattgccagatatctacatcccaaaccacttgtcattgcacgattacatcttcc aaaacatctctgaattcgctaccaagccatgtttgattaacggtccaactggtcatgtttacacctactc tgatgttcacgttatctccagacaaattgctgctaactttcacaagttgggtgtcaatcaaaacgatgtc gtcatgttgttattgccaaactgtccagaattcgtcttgtctttttggctgcttcttttagaggtgctactgct acagctgctaatccatttttactccagctgaaattgctaagcaagctaaggcttctaacaccaagttg attattaccgaagctagatacgttgacaagatcaagccattgcaaaatgatgatggtgttgttatcgtct gcatcgatgataatgaatccgttccaattccagaaggttgtttgagattcactgaattgactcaatctac taccgaagcctccgaagttattgattccgttgaaatttctccagatgatgttgttgctttgccatactcttca ggtactactggtttgccaaaaggtgttatgttgactcataagggtttggttacatccgttgctcaacaagt tgatggtgaaaatccaaacttgtacttccactccgatgatgtcatttgtgtgtttgccaatgttccatatc tacgccttgaactctattatgttgtgcggtttgagagttggtgctgctatttgattatgccaaagttcgaaa tcaatttgttgttggaattgatccaaagatgcaaggttactgttgctccaatggttccaccaatagttttgg ctattgctaagtcctctgaaaccgaaaagtacgacttgtcctctatcagagttgttaagtcaggtgctgc tccattgggtaaagaattagaagatgctgttaacgccaagttcccaaatgctaaattgggtcaaggtt 
acggtatgactgaagctggtccagtttagctatgtctttgggttttgctaaagaaccattcccagtaaa atctggtgcttgtggtacagttgttagaaacgctgaaatgaagatcgttgatccagatactggtgactc cttgtctagaaatcaaccaggtgaaatctgcatcagaggtcatcaaattatgaagggttacttgaaca atccagctgctactgcagaaaccattgataaggatggttggttgcatacaggtgatattggtttgattg atgacgacgacgaattattcatcgttgatagattgaaagaattgatcaagtacaagggtttccaagtt gctccagcagaattggaagctttgttgattggtcatccagatattaccgatgttgctgttgttgcaatgaa ggaagaagctgctggtgaagttccagttgctttgttgtcaaatccaaggactctgaattgtccgaaga tgatgtaaagcaattcgtcagtaagcaagtcgtttctacaagagaatcaacaaggtttcttcaccga atccattccaaaagctccatctggtaagatcttgagaaaagatttgagagccaagttggctaacggtt tgtga

CbHPPR atggaagccatcggtgtttgatgatgtgtccaatgtctacctacttggaacaagaattggacaagag attcaagttgttcagatactggactcaaccagctcaaagagatttttggctttacaagccgaatccatt agagctgttgttggtaattctaatgctggtgctgatgccgaattgattgatgcttgccaaaattggaaat cgtttcctcttctctgttggtttggataaggtcgatttgatcaagtgtgaagaaaagggtgttagagttac caataccccagatgtttgacagatgatgttgctgatttggccattggtttgattttggctgtttgagaag aatctgcgaatgcgataagtacgttagaagaggtgcttggaaattcggtgatttaagttgactacca agttctccggtaagagagttggtattattggtttgggtagaatcggtttagctgttgctgaaagagctga agcttttgattgtccaatctcctacttctccagatctaaaaagccaaacactaactacacctactacggt tctgttgttgaattggcttccaactccgatattttggttgttgcttgtccattgactccagaaactacccatat cattaacagagaagttatcgatgctttgggtcctaagggtgtcttgattaacattggtagaggtccacat gttgacgaaccagaattggtttctgctttggttgaaggtagattgggtggtgctggtttagatgtttcgaa agagaaccagaagtcccagaaaagttgttcggtttggaaaacgttgtttgttgcctcatgttggttctg gtactgttgaaactagaaaggttatggctgacttggttgtcggtaatttggaagctcattttctggtaag cctttgttgacaccagttgtctga

CDRAS atgaagatcgaagttaaggactccaccatgattaagccatctgctgaaactccaggtggttcttgtg gttgtctaatttggatttgttgtccccagctaactaccatactttgtctgttcatttctactcccacgatggttc tgataatttcttgatgctgccggtttgaaagaatccttgtctagagctttggttgaattctatccatacgct ggtagattgaagttgaacggtaacagattggaaatcgactgtaacaacgaaggtttgttgttggttga agctgaatgtgatggtgcattggatgaattgggtgattttgctccaagaccagaattgaacttgatccc aaaagttgactactccagaggtatttctacttacccattgatggtttccaattgaccagattcaaatgtg gtggtgttgctttgggtgttgctaatgaacatcatttgtctgatggtgttgcagccttgcatttatcaatactt gggctcatttgtcaagaggtgctccagctccaactccattgccacattttgatagatcatcttgtctgct agaaacccaccacaacctcaattttctcatgctgaatatcaaccaccaccaaccttggaaaatccat taccacatactgatatcgcccactctagattcaagttaaccagagatcaattgaactccttgaagtcc aagtttaaaacagctccagctgatggtggtgctggtaaatcttattctactttcgaagtttggccggtca catttggagatctgtatgtattgctagaggtttgccagaaggtcaagaaactaagttgcatattccattc gatggtagaggtagattgcaattgccaccaggttttttggtaacgctattttcttcgctactccaattgct acctgtggtgaaattgaatccaactctttgaactacgccgttagaagagtttcagatggtgtttccagat tagacgaagattacttgagatcctccatcgatttcttagaattgcaagaagatatctccaagttggctc aaggtgctcattcttttagatgtccaaacttgtgggttatctcttgggtttggttgccaatctatgaaccag 
atttggttggggtaaagctg tttacatgggtccatgggctgctccatttgaaggtaaatcatatttgttgc caaacccagaaaaggacggttccttgtttgtttctattaccttgcataagcaacacatggaaagattc gaaaagttgttctacgaaatttga

MORAS atgagaatcgacatcaaggattccaccatggttaagccagctgctgaaactccaggtggttctgttg gttgactaatttggatttgttgtccccagctaactaccatacttgtctgttcatttctaccaccacgatggtt ctgaaaatttctttgatgctgctgctttgaaagaagctttgtctagagctttggttgatttctatccatacgct ggtagattgaagttgaaggacaacagattggaaatcgattgcaatggtgaaggtgtttgttggttga agctgaatctgatggtgcttggctgaattgggtgaatttgctccaagaccagatttgaatttgatccca caagttgattacgccaagggtatttctacttacccattgatgttgttccaattgaccagattcaaatgtgg tggtgttggtttgggtgttgctaatgaacatcatttgtcagatggtgttgctgccttgcatttatcaatactt gggctcatttggctagaggtgttccagctccatctccaccaccagttttgatagaagatcttgtctgct agaaacccaccaaagccacaattttctcatgctgaatatcaaccaccaccaactttgccaactccat tgactgatactgctattgcttactccaaattgaaggtcaccagagatcaattgggtgcttgaaggcta aatgtttggctggtgatccatctggtaaacctagatctactttgaagtttggccggtcatatttggagat gtgtttgtgctgcaagaggtttgccagaagatcaagaaactaagttgcatatcccattcgatggtaga gctaaattgagattgccaccaggttattttggtaacgctattttcttgctactccagttgctacctgtggtg aaattgaatctaattcttggctcacgccgttaagagagttggtgatgctattgcaagattggacgaag attacttgagatcctccatcgatttcttagaattgcaagaagatatctccaagttggctcaaggtgctca ttcttttagatgtccaaatttgtgggttatctcctgggttagattgccagtttatgaaccagattttggttggg gtaaagctgtttacatgggtccatgggctgctccattcgaaggtaaatcttatttgttgccaaacccag ataacgacggttcttgtttgttgctattaccttgcatacccaacacatggaaagattcgaaaagttgttc tacgaaatttga

SmCYP98 atggctgctttgttgttggctccagttgctgctgtttggctgttgtcttgtatcatatctactacagattgaga A14 ttcagattgccaccaggtccatttccatggccagttgttggtaacttgtatgatattaagccagtcagatt cagatgctttgctgaatgggctcaatcttatggtccaatttgtctgtttggttcggttctactttgaacgttgt tgtctctaactccgaattggccaaagaagtcttgaaagaaaaggatggtcaattggccgatagaca cagatcaagatctgctgttaagttgtctagagatggtcaagatttgatttgggctgattacggtccacatt acgttaaggttagaaaggtctgtaccgtcg ttttgtttctccaaagagattggaattgttaagaccaatc agagaagatgaaatcaccgccatgg ttgaatctatctacaatgattctactgcctcctctggtaagtct gttgtttgaaaaagtacttggcttccatggctttccataacattaccagattggttttcggtaagagattc gtcaattctgaaggtgctgttgataagcaaggtcaagaattcaaagccattgccattaacggtttgaa attgggtgcttctttagctatggctgaacatattccatggttaagatgggctttccattggatgaagatgc tttactcaacatggtgccagaatggaaagattgaccagagaaattatgcaagaacacaccttgttg agacaaaaaactggtggtgctaagaaccatttcttgatgctttgttaaccttgaaggacgaatacgat ttgtccgaagataccattattgccttgttgtgggatatgattgctgctggtatggatactccagctatttctg ttgaatgggccatggctgaattggttagaaatccaagagttcaacaaaaggttcaagaagaattgg acagagttatcggtagagatagagttatgaccgaagttgatattccaaacttgccatacttgcaatgc gtcgtcaaagaaagtttgagattgcatccaccaactccattgatgttgccacatagagctaacacca atgttgaaatcggtggttacgatattcctaagggttccaacgttaatgttaatgtttgggccgttgctaga gatccagctgtttggaaaaatccattggaattcagaccagaaagattcgtcgaagaaggtattgaca 
ttaagggtcacgatttcagagtttgccatttggtgctggtagaagagtttgtccaggtgctcaattgggt attgatttgactacatccatgatcggtcatttgttgcatcattttcttgggctccaccagcaggtatgaga actgaagaaatcaatttggacgaaaacccaggtactgttacctacatgaagaatccagttgaagctt tgccaactccaagattggctccacacttgtacaaaagagttgctgttgacaccatctga

$S m C P R \quad$ atggaaccatcctctaaaaagttgtccccattggatttcattaccgccatttgaagggtgatattgaag gtgttgctccaagaggtgttgcagctatgttgatggaaaacagagatttggctatggtttgactacctct gttgctgtttgattggttgcgttgttgtttggcttggagaagaactgctggttctgctggtaaaaaacaat tgcaaccaccaaagttggttgttccaaaaccagctgctgaacctgaagaagctgaagacgaaaa aactaaggtcagtgtttcttcggtactcaaactggtactgctgaaggtttgctaaagctttgccgaag aagctaaagctagatatccacaagctaagttcaaggttatcgatttggatgattacgctgccgatgat gatgaatacgaagaaaagttgaagaaagaatccttggccttcttcttcttggcttcttatggtgatggtg aacctactgataatgctgctagattttacaagtggttcaccgaaggtaaggatagagaagattggttg aagaacttgcaatacggtgttttggtttgggtaacagacaatacgaacacttcaacaagattgccat cgttgtcgatgatttgattactgaacaaggtggtaagaagttggttccagttggtttaggtgatgatgatc aatgcatcgaagatgattttccgcttggagagaattggtttggccagaattggataagttgttgagaa atgaagatgatgctactgttgctactccatataccgctgttgttttacaatacagagttgtcttgcacgat caaactgatggtttgatcacagaaaatggttctccaaatggtcatgctaacggtaacactatctatgat gctcaacatccatgtagagctaacgttgctgttagaagagaattgcatactccagcttcagatagatc ttgtacccatttggaattcgatacttcaggtactggtttggtttacgaaactggtgatcatgttggtgtttact gcgaaaacttgttggaaaatgtcgaagaagccgaaaagttattgaacttgtctccacaaacctactt ctccgttcatactgataacgaagatggtactccattgtctggttcttcattgccaccaccatttccaccat gtactttgagaactgctttgactaagtacgccgatttgatttctatgccaaagaagtctgtttggttgcttt ggctgaatacgcctctaatcaatcagaagctgatagattgagatacttggcttcaccagatggtaaa gaagaatacgcccaatatatcgttgcctcccaaagatcattattggaagttatggctgaattcccatct gctaaaccaccattgggtgtttttttgctgctattgctcctagattgcaacctagattctactccatttcttc ctccccaaaaattgctccaactagagttcatgttacctgtgctttggtttatgataagactccaactggta gaatccataagggtatttgttctacctggattaagaacgctgttccattggaagaatcttcagattgctct tgggctccaatttcatcagaaactctaactttaagttgccagccgatccaaaggttccaattatcatgg ttggtccaggtacaggtttagctcctttagaggtttcttacaagaaagattggccttgaaagaatctggt gctgaattgggtccagctattttgtttttggttgtagaaacagaaagatggacttcatatacgaagatg aattgaactccttcgttaaggttggtgccatttctgaattgatcgttgcttttctagagaaggtccagcca aagaatacgttcaacataagatgtctcaaagagcctccgatatttggaagatgatatctgatggtggt tacatgtacgttgtggtgatgctaaaggtatggctagagatgttcatagaaccttgcataccattgctc aagaacaaggttctttgtcatcttctgaagcagaaggtatggtcaaaaacttgcaaactactggtaga tactgagagatgtctggtga

CbCYP98 atggcttcccctttgttgatttgttctcttgccattggctgttgtcttgtaccatttgttctacagattgagata A14 cagaattccaccaggtccaagaccatggccagttgttggtaacttgtatgatgttaagccagtcagat tcagatgttttgctgaatgggctcaattattcggtccaacttttctgtttggttcggttctactttgaacgtca tcgtttcttcatccgaattggccaaagaagtcttgaaagaaaaggatggtcaattggccgatagaca cagatcaagatctgctgttaagttgtctagagatggtaaggatttgatttgggctgattatggtccacac 
tacgttaaggttagaaaggtctgtaccgtcgtttgtttctccaaagagattggaattgttaagaccaat cagagaagacgaaatcaccgttatggttgaatccatctaccaagattctgctgcttctggtaaatccgt tgtcatcaaaaagtacttggcttctatggccttccataacattaccagattggttttcggtaagagattcg tcaattccgaaggtgaagttgataagcaaggtcaagaattcaaggccattgctattaacggtttgaa attgggtgcttccttggctgtttctgaacatattccatggttgagatggatgttcccattggatgaagatgc tttactcaacacggtgtcagaatggaaagattgaccagagaaatcatgcaagaacataccttggct agacaaaaaactggtggtgctaagcaacatttcttcgatgctttgttgaccttgaaggatgaatacgat ttgtccgaagataccattattgccttgttgtgggatatgattgctgctggtatggatactccagctatttctg ttgaatgggcaatggctgaattggttagaaatccaagagttcaagaaaaggttcaagaagaattgg acagagttatcggtcacgaaagaattatgaccgaattggatattccaaacttgccatacttgcaatgc gtcgtcaaagaatcattgagattgcatccaccaactccattgatgttgccacatagatctaatgccgat gttaagattggtggttacgatatcccaaagggttctaacgttcatgttaacgtttgggctattgctagag atccaaaatcttggaaagacccattggaattcagaccagaaagattcttggaagaagatgttgacat caagggtcacgactttagattattgccatttggtgctggtagaagagtttgtccaggtgctcaattgggt attgatttggctacatctatgatcggtcatttgttgcatcatttcagatggactcctccagctggtgttaga gctgaagatattgatatgggtgaaaacccaggtactgttacttatatgagaactccagttgaagctgtt ccaactccaagattgccagctgacttgtacaaaagagttgccgttgttgatatttga

$C b C P R$ atggaatccaccatcgaaaaattgtccccattggatttgatgaccgctatttgaaaggtgtcaagttg gataactctaacggttctgctggtgttgaacatccagctgttgttgctatgttgatggaaaacaaagact tgatgatgatgttgaccacctctgttgctgttttgttgggtttggttgtttacttggtttggagaagaggtgct ggttctgctaaaaaagttgttgaaccacaaaagttggttgtcccaaaaggtccagttgaagttgaag aagttgacgacggtaaaaagaaggttaccatttcttcggtactcaaactggtactgctgaaggttttg ctaaagctttggctgaagaagctaaagctagatatccacaagctaacttcaaggttgttgatatggat gattacgctgccgatgatgaagaatacgaagaaaagatgaagaaagaaaccttcgccttcttcttct tggctacttatggtgatggtgaacctactgataatgctgctagattttacaagtggttctccgaaggtaa agatagaggtgatatgttcaagaacttgcactacggtgttttggtttgggtaacagacaatacgaac acttcaacaagattgccatcgttgtcgatgatttgttgtttgaacaaggtggtaagagattggttccagtt ggtttaggtgatgatgaccaatgtatcgaagatgactttccgctagaagagataatgtttggccaga attggacaacttgttgagagatgaagattacccaccagtttctactccatatactgctgctgttttagaat acagagtcgttttccacgatcaatccgatgaattgccatctgaaaacactttggctaatggtagagct aatggtcatgcttcttatgatgctcaacatccatgtaaggttaacgttgccgttaagaaagaattgcata ccccattgtctgatagatcttgtacccatttggaattcgacatttctggtactggtttggaatacgaaaca ggtgatcatgttggtgtttactgcgaaaacttgatcgaaactgtagaagaagccgaaagattattggg tttgtctccacaaactttcttctctgttcataccgacaaagaagatggtactccattgggtggttcagcttt gccaccaccatttccaccatgtactttgagaactgcttgactagatacgctgatttgttatctgctccaa aaaaggctgctttgattgctttagctgcttatgcttctgatcctgttgaagctgaaagattgactcatttggt ttctccagctggtaaagaagaatacgctcaatacgttgttgctggtcaaagatccttgttggaagttatg gctgattttccatctgctaaaccacctttgggtgtttttttgctggtattgctccaagattgcaacctagatt ctactctatttcctcctccccaaaaattgctccatccagaattcatgttacctgcgctttggtttacgaaaa aactccaactggtagaatccataagggtgtttgttctacttggatgaagaacgctgttccattggaaga 
atctccaaactgttcttcagctccagttttcgttagaacctctaatttagattgccagccgatccaaagg ttccagtaattatgattggtccaggtacaggtttggctccttttagaggtttttacaagaaagattggcctt gaaagaatccggtgctgaattgggtccagcagttttgtttttggttgcagaaactccaagatggacttc atctatcaagacgaattggataatttcgttcaagccggtgttgtttccgaattggtttggcttttctagag aaggtccagccaaagaatatgtccaacataagatggctcaaaaggcttgggatgtttggaacatga tttctgaaggtggttacgtttacgtttgtggtgatgctaaaggtatggctagagatgttcatagaacctta cacaccattgtccaagaacaaggttcattggattcttctaagaccgaatccttcgtcaaaaacttgca aatgactggtgcagtcttaagagatgtttggtga

EchpaB atgaagccagaagatttcagagcctctactcaaagacctttactggtgaagaatacttgaagtcctt gcaagatggtagagaaatctacatctatggtgaaagagttaaggacgttactactcatccagctttta gaaatgctgctgcttctgttgctcaattatacgatgcattgcataagccagaaatgcaagattctttgtgt tggaacactgatactggttctggtggttacactcataagttttcagagttgctaagtccgccgatgattt gagacaacaaagagatgctattgccgaatggtctagattgtcttatggttggatgggtagaactcca gattacaaagctgctttggttgtgctttgggtgctaatccaggtttttatggtcaattcgaacaaaacgct agaaactggtacaccagaattcaagaaacaggtttgtacttcaaccacgctatagttaacccacca atcgatagacatttgccaactgataaggttaaggatgtctacatcaagttggaaaaagaaaccgat gccggtattatagtttctggtgctaaagttgttgctaccaactctgctttgactcactacaatatgattggtt tcggttccgctcaagttatgggtgaaaatccagattttgccttgatgtttgttgctccaatggatgctgatg gtgttaagttgatttctagagccagttacgaaatggttgctggtgctacaggttctccatatgattatccat tgtcctctagattcgacgaaaacgatgctatcttggttatggataacgtttgatcccttgggaaaacgt cttgatctacagagattttgacagatgtagaagatggactatggaaggtggtttgctagaatgtatcc attgcaagcttgtgttagattggcagttaagttggacttcattaccgccttgttgaaaaagtctttggaat gcactggtactttggaattcagaggtg ttcaagctgatttgggtgaagttgtagcttggagaaatactttt tgggccttgtctgattctatgtgttctgaagctactccatgggttaatggtgcttatttgccagatcatgctg cattgcaaacttacagagttttggctcctatggcttacgctaagattaagaacatcatcgaaagaaac gtcacctccggtttgatctatttgccatcttctgctagagatttgaacaacccacaaattgaccaatactt ggctaagtacgttagaggttctaacggtatggatcacgttcaaagaatcaagatttgaagttgatgtg ggatgccatcggttctgaatttggtggtagacatgaattatacgaaatcaattactccggttcccaaga cgaaattagattgcaatgtttaagacaagcccaatcctctggtaacatggataagatgatggctatgg ttgatagatgcttgtccgaatacgatcaaaatggttggactgttccacacttgcataacaacgatgac attaacatgttggacaagttgttgaagtaa

EchpaC atgcaattggacgaacaaagattgagattcagagatgctatggcttctttgtctgctgccgttaacatta ttactactgaaggtgatgctggtcaatgtggtattactgctactgctgttgttctgttactgatactccacc atcttgatggtctgtattaacgctaattctgccatgaacccagttttcaaggtaacggtaaattgtgcgt taacgtcttgaaccacgaacaagaattgatggctagacatttgctggtatgactggtatggctatgga agaaagattctctttgtcttgttggcaaaaaggtccattggctcaaccagtttgaaaggttctttagcttc tttggaaggtgaaatcagagatgttcaagccattggtactcacttggtttacttggttgaaatcaagaa catcatcttgtccgctgaaggtcatggtttgatctactttaagagaagattccacccagttatgttggaa atggaagctgctatttga 


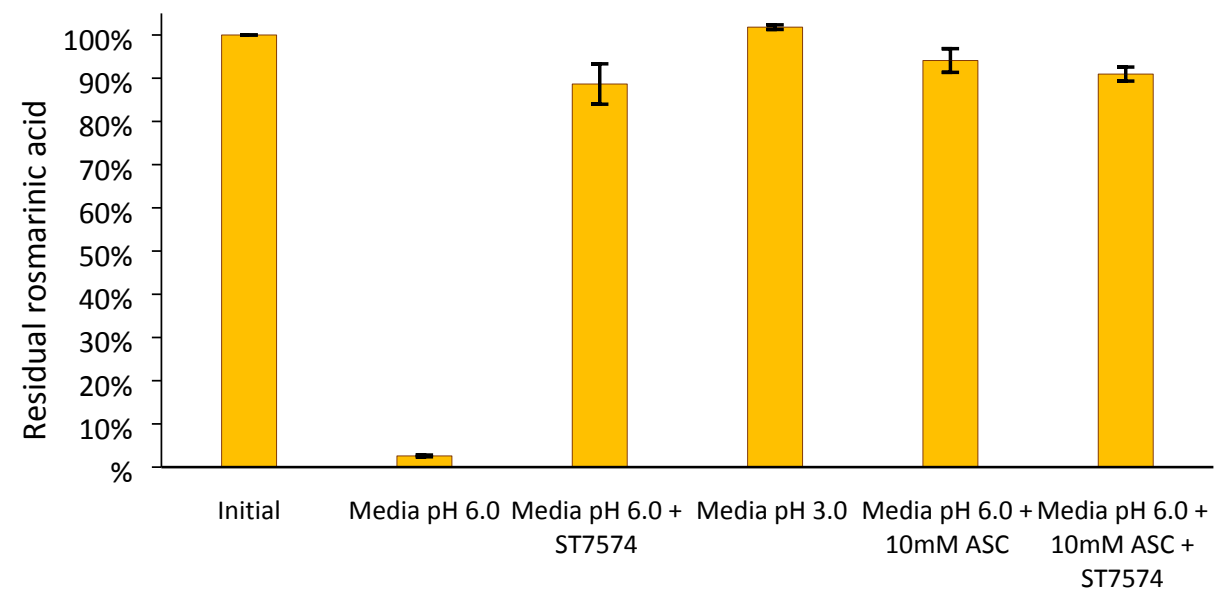

Supplementary Figure S1: Stability of rosmarinic acid in mineral medium. Rosmarinic acid is supplemented in mineral media followed by incubation for 72 hours at $30^{\circ} \mathrm{C}$. The degradation is extremely $\mathrm{pH}$ dependent. Supplementing the media with $10 \mathrm{mM}$ ASC (ascorbic acid) as antioxidant agent can significantly stabilize RA in the media. ST7574 is the parental strain used in the strain construct. The values represent the mean of $n=3$ biologically independent samples and error bars show standard deviation.

In this step, we aimed to investigate rosmarinic acid stability in culture medium. To do this, we supplemented $1 \mathrm{~g} / \mathrm{L}$ of RA to mineral media (at pH 6.0), and observed for RA content after 72 hours of incubation at $30^{\circ} \mathrm{C}$, with and without inoculation of parental strain ST7574, as shown in Supplementary Figure S1. To our surprise, almost $98 \%$ of RA in the media at $\mathrm{pH} 6.0$ was depleted, while this value for inoculated media at $\mathrm{pH} 6.0$ was $3 \%$. In other words, the growth of cells has preserved RA against degradation, which was probably due to $\mathrm{pH}$ drop of yeast culture, to almost $\mathrm{pH}$ 4.0 at the end of 72 hours. To verify this, we repeated the same experiment with mineral media at $\mathrm{pH} 3.0$, and we did not observe any decrease in RA content after 3 days of incubation at $30^{\circ} \mathrm{C}$ (Supplementary Figure S1), which highlighted the importance of $\mathrm{pH}$ on rosmarinic acid stability. However, as cultivating the cells at low pH results in a significant stress on cells, we investigated inhibition of spontaneous degradation by supplementing reducing agents, namely ascorbic acid (ASC) to the media and culture. The results showed $94 \%$ and $91 \%$ of initial RA was retrieved at the end of incubation for the media with $10 \mathrm{mM} \mathrm{ASC}$, with and without strain growing, respectively. Hence, in all the RA production tests we used mineral media at pH 6.0, supplemented with $10 \mathrm{mM}$ ASC. 
(A)

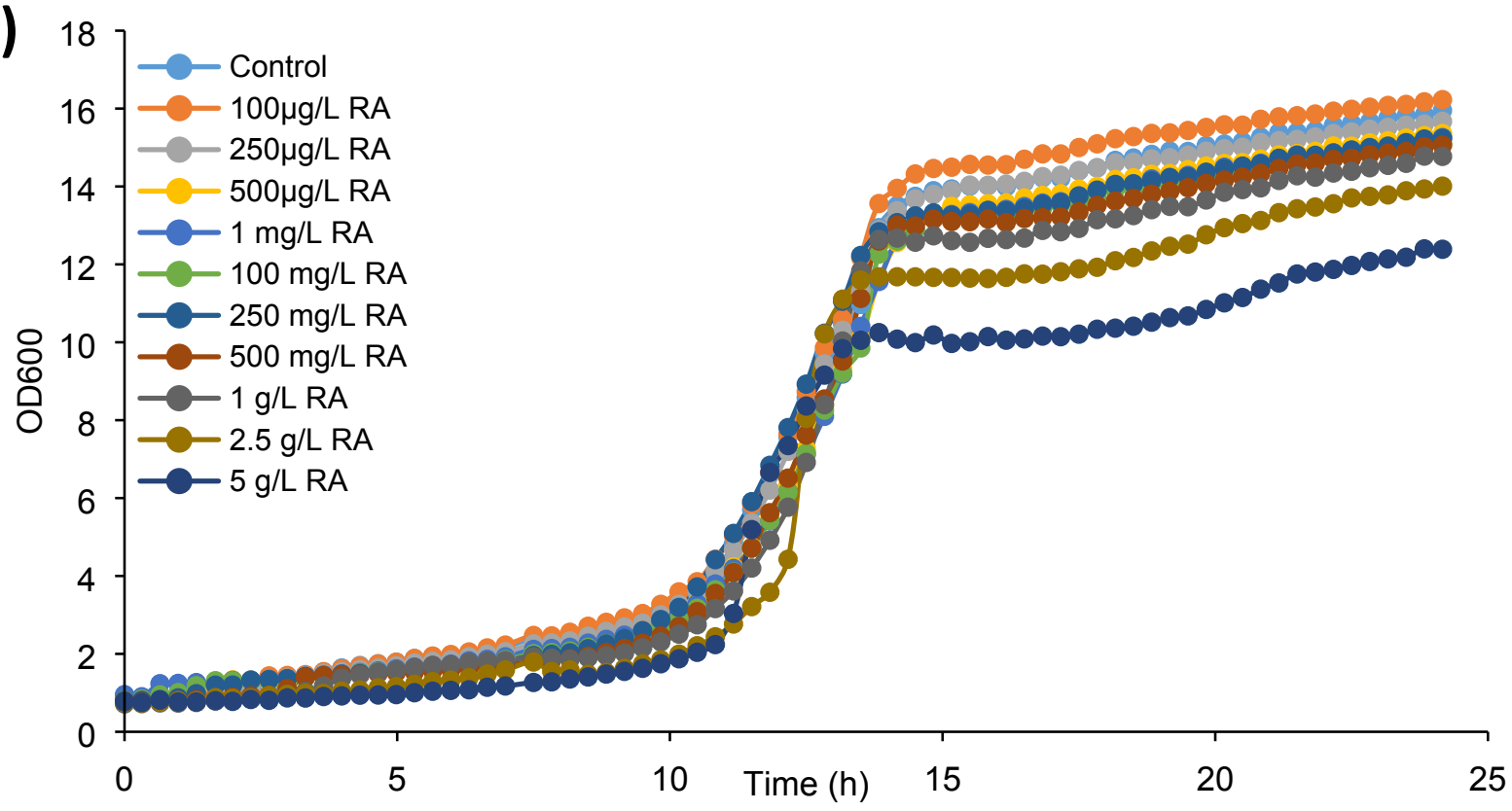

(B)

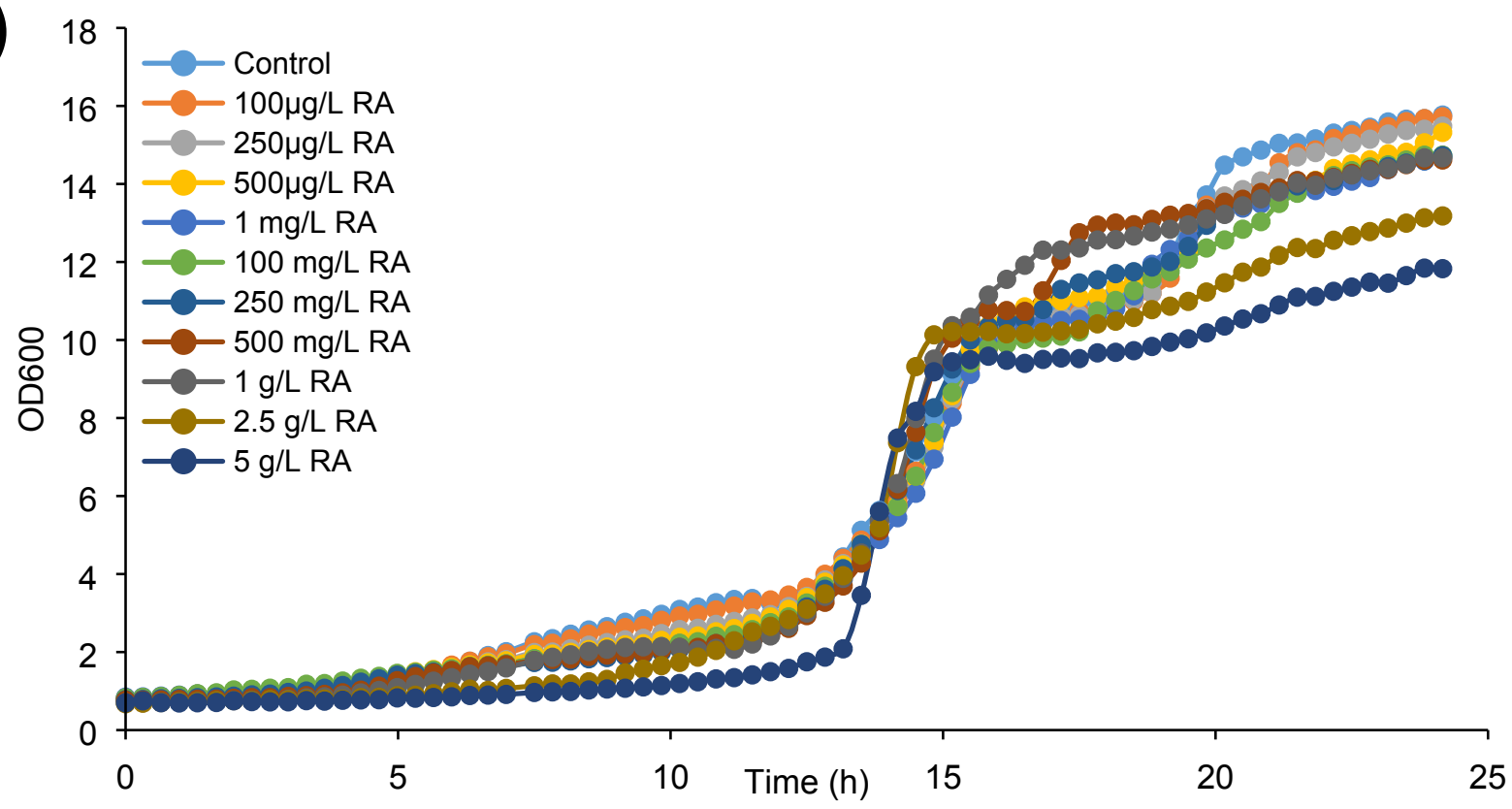

Supplementary Figure S2: Toxicity test of rosmarinic acid on growth of yeast strains. (A) ST7574; parental strain, and (B) ST9411; strain with integrated pathway for RA production. No lethal effect of RA up to $5 \mathrm{~g} / \mathrm{L}$ was observed on yeast. Final measurement of RA in cultures showed no significant change in rosmarinic acid concentration, showing that there was no cell uptake of RA. 
(A)

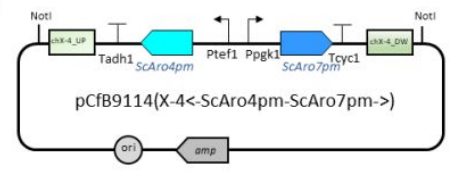

(B)

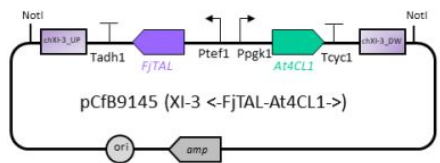

(C)

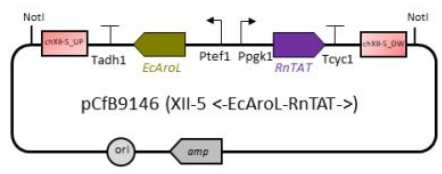

(D)

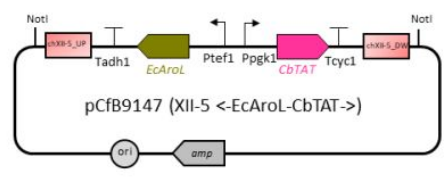

(E)

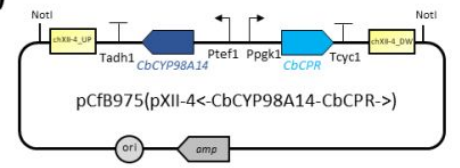

(F)

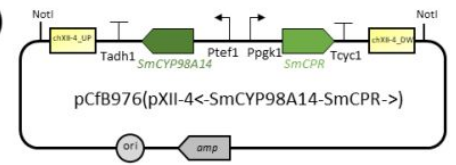

(G)

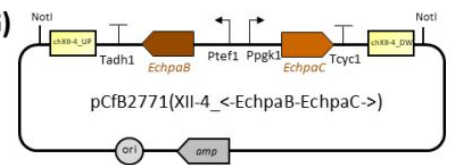

(H)

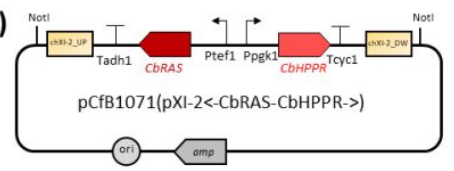

(I)

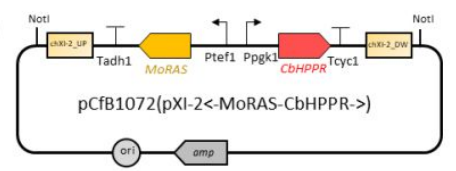

(J)

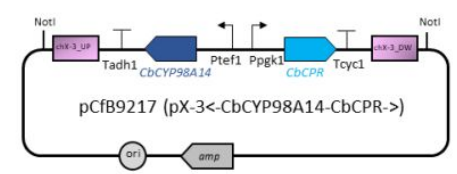

(K)

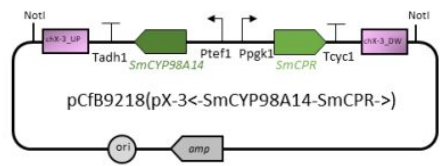

(L)

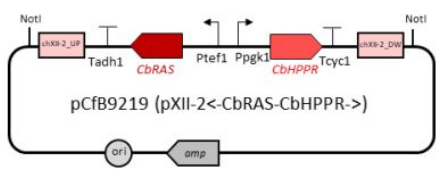

(M)

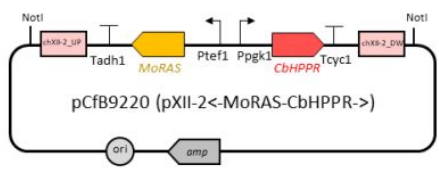

(N)

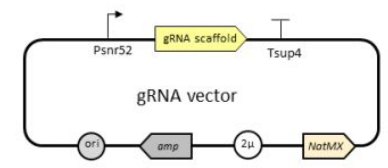

Supplementary Figure S3: Plasmid maps of integrative vectors. All the integration fragments contain expression cassettes flanked with $\sim 500$ bp long integration regions, i.e. shown as chX-4_UP and chX4_DW in (A). The plasmids also contain a Notl restriction site, that get linearized by Notl digestion. The exact loci of EasyClone sites is explained in Jessop-Fabre et al., $2016^{6}$.For integration of each of the plasmids $A-M$, the corresponding gRNA vector with the map shown in $(\mathrm{N})$ is used. The correct integration is then verified by colony PCR, as described in "Strain and media" section in manuscript. 
(A)<smiles>N[C@@H](Cc1ccc(O)cc1)C(=O)O</smiles>

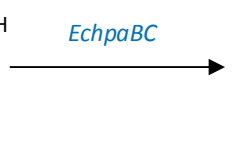<smiles>N[C@@H](Cc1ccc(O)c(O)c1)C(=O)O</smiles>

L-DOPA

L-(3,4-dihydroxy)phenylalanine

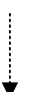

Melanin

(B)

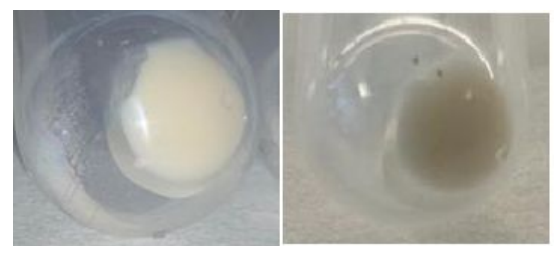

Parent strain Chimeric strain\#2

(ST7574) (ST9414)

Supplementary Figure S4: L-tyrosine degradation to melanin by hydroxylase enzyme EchpaBC. (A) Enzymatic pathway of L-tyrosine hydroxylation to L-DOPA and then spontaneous oxidation to darkcolored pigment melanin. The dashed arrow shows non-enzymatic, spontaneous oxidation pathway. (B) The dark color of chimeric strain \#2 compared to parent strain at the end of 3 days of cultivation. 


\section{Supplementary References:}

(1) Bloch, S. E., and Schmidt-Dannert, C. (2014) Construction of a chimeric biosynthetic pathway for the de novo biosynthesis of rosmarinic acid in Escherichia coli. Chembiochem 15, 2393-2401.

(2) Li, Z., Wang, X., and Zhang, H. (2019) Balancing the non-linear rosmarinic acid biosynthetic pathway by modular co-culture engineering. Metabolic Engineering 54, 1-11.

(3) Milne, N., Tramontin, L. R. R., and Borodina, I. (2020) A teaching protocol demonstrating the use of EasyClone and CRISPR/Cas9 for metabolic engineering of Saccharomyces cerevisiae and Yarrowia lipolytica. FEMS Yeast Res. 20.

(4) van der Hoek, S. A., Darbani, B., Zugaj, K. E., Prabhala, B. K., Biron, M. B., Randelovic, M., Medina, J. B., Kell, D. B., and Borodina, I. (2019) Engineering the Yeast Saccharomyces cerevisiae for the Production of L-(+)-Ergothioneine. Front Bioeng Biotechnol 7, 262.

(5) Stovicek, V., Borodina, I., and Forster, J. (2015) CRISPR-Cas system enables fast and simple genome editing of industrial Saccharomyces cerevisiae strains. Metabolic Engineering Communications 2, 13-22.

(6) Jessop-Fabre, M. M., Jakočiūnas, T., Stovicek, V., Dai, Z., Jensen, M. K., Keasling, J. D., and Borodina, I. (2016) EasyClone-MarkerFree: A vector toolkit for marker-less integration of genes into Saccharomyces cerevisiae via CRISPR-Cas9. Biotechnol J 11, 1110-1117.

(7) Rodriguez, A., Kildegaard, K. R., Li, M., Borodina, I., and Nielsen, J. (2015) Establishment of a yeast platform strain for production of $\mathrm{p}$-coumaric acid through metabolic engineering of aromatic amino acid biosynthesis. Metab. Eng. 31, 181-188. 